Differentialdiagnose. Von der Krankheit abzugrenzen sind nicht mit dem Gallengangsystem zusammenhängende Zysten.

Therapie. Folgende Möglichkeiten stehen zur Verfügung: Antibiotika zur Behandlung der Cholangitis, endoskopische Gallengangdrainage, ERCP mit extrakorporaler Stoßwellenlithotrypsie, interne Gallengangdrainage, Hemihepatektomie oder Lebertransplantation in ausgewählten Fällen.

Prognose. Im Verlauf der Krankheit kann es zu rezidivierenden Cholangitiden kommen, zu Cholezysto- bzw. Cholelithiasis, zu Leberzirrhose mit portaler Hypertension und in $7 \%$ der Fälle zu Cholangiokarzinomen.

\section{Literatur}

Asselah T, Ernst O, Sergent G, L'Hermine C, Paris JC (1998) A magnetic resonance cholangiopancreatography diagnosis. Am J Gastroenterol 93: 109-110
Caroli J, Corcos V(1964) La dilatation congenitale de voies biliaires intrahepatiques. Rev Med Chir Mal du Foie 39: 1-70

Dagli U, Atalay F, Sasmaz N, Bostanoglu S, Temucin G, Sahin B (1998) Caroli's disease: 1977-1995 experience. Eur J Gastroenterol Hepatol 10: 109-112

Gold DM, Stark B, Pettei MJ, Levine JJ (1995) Successful use of an internal biliary stent in Caroli's disease. Gastrointest Endosc 42: 589-592

Keane F, Hadzic N, Wilkinson ML, Qureshi S, Reid C, Baker AJ, MieliVergani G (1997) Neonatal presentation of Caroli's disease. Arch Dis Child 77: F145-F146

Pinto RB, Lima JP, Silveira TR da, Scholl JG, Mello ED de, Silva G (1998) Caroli's disease: report of 10 cases in children and adolescents in Southern Brazil. J Pediatr Surg 33: 1531-1535

Sans M, Rimola A, Navasa M et al. (1997) Liver transplantation in patients with Caroli's disease and recurrent cholangitis. Transpl Int 10: 241-244

Taylor ACF, Palmer KR (1998) Caroli's disease. Eur J Gastroenterol Hepatol 10: 105-108

Torra R, Badenas C, Darnell A, Bru C, Escorsell A, Estivill X (1997) Autosomal dominant polycystic kidney disease with anticipation and Caroli's disease associated with a PKD1 mutation: rapid communication. Kidney Int 52: 33-38

\title{
128 Fulminantes Leberversagen und Lebertransplantation
}

\author{
B. Rodeck, M. Melter
}

\subsection{Fulminantes Leberversagen}

\section{B. Rodeck, M. Melter}

Definition. Ein fulminantes (oder: akutes) Leberversagen liegt vor, wenn eine akute Leberkrankheit bei einem vorher Lebergesunden innerhalb von 8 Wochen zu einer hepatischen Enzephalopathie führt. Für das Kindesalter ist diese Definition nur bedingt anzuwenden. Eine gering- bis mäßiggradige Enzephalopathie ist im Neugeborenen- und Säuglingsalter kaum zu diagnostizieren. Das Ausmaß der Leberfunktionseinschränkung ist daher im Kindesalter mit $\mathrm{zu}$ berücksichtigen [Quicktest $<40 \%$, Cholinesteraseaktivität $<2,5 \mathrm{kU} / 1$ vor Substitution von Gerinnungsfaktoren bzw. FFP (fresh frozen plasma)]. Für die Pädiatrie sollte daher der Begriff „fulminantes Leberversagen“ wie folgt definiert werden: Anamnese nicht länger als 6 Monate, vorher lebergesund (Ausnahme Neugeborene), Einschränkung der Lebersyntheseleistung (s. oben) mit und ohne hepatische Enzephalopathie.

Epidemiologie. Genaue Angaben über die Häufigkeit des fulminanten Leberversagens (FLV) im Kindesalter existieren nicht. In den USA wird insgesamt eine Inzidenz von $1: 115000$ Einwohner pro Jahr angegeben mit einer Letalität von annähernd $80 \%$.

Ätiologie. Die häufigsten Ursachen eines FLV im Kindesalter sind Infektionen und Intoxikationen. In verschiedenen $\mathrm{Al}-$ tersstufen überwiegen bestimmte Ursachen. In Tabelle 128.1 sind die wesentlichen Ursachen für die Neonatalzeit, für Säuglinge und Kleinkinder bis zu einem Alter von 3 Jahren und für ältere Kinder aufgeführt.
Pathogenese und Pathologie. Das auslösende Agens schädigt primär die Hepatozyten. Die Faktoren, die das Ausmaß der Leberzellschädigung bedingen, sind nicht genau bekannt. Bei bestimmten Infektionen spielt das Alter, bei der Bewältigung der Infektionen der Immunstatus, bei Intoxikationen ein genetisch-biochemischer Polymorphismus eine wesentliche Rolle. Bei den meisten Fällen mit FLV liegt histologisch eine Leberzellnekrose vor. Bei Virusinfektionen wirkt entweder das Virus selbst zytotoxisch, oder es induziert eine zytopathogene Immunantwort. Das toxische Agens bei Intoxikationen oder Stoffwechselkrankheiten kann meist identifiziert werden. Als Ausdruck der hohen Regenerationskapazität der Leber werden beim FLV erhöhte Spiegel des humanen Hepatozytenwachstumsfaktors (hHGF) gemessen. Auf der anderen Seite werden auch Inhibitoren der Zellregeneration aus den nekrotischen Hepatozyten freigesetzt. Der Ausgang eines FLV nach der Elimination des auslösenden Agens wird deshalb durch das Überwiegen der regenerativen oder der inhibierenden Faktoren bestimmt. Überwiegen die regenerativen Faktoren, kommt es zu einer Restitutio ad integrum; überwiegen die inhibierenden Faktoren, kommt es zum kompletten Leberausfall.

Klinische Symptome. Nach unspezifischen Krankheitssymptomen entwickelt sich ein Ikterus. Selten zeigt sich eine Enzephalopathie schon vor dem Auftreten des Ikterus. Der Ikterus sowie Anorexie, Erbrechen, Blutungen, später Foetor hepaticus, Aszites und die Zeichen der hepatischen Enzephalopathie ermöglichen die klinische Diagnose des FLV. Laborchemisch finden sich eine Hyperbilirubinämie und eine Erhöhung der Transaminasen (GOT, GPT, GLDH). Fallende oder niedrige Transaminasen sind insbesondere bei abnehmender Lebergröße (Sonographie) ein Zeichen der bereits abgelaufenen massiven Leberzellnekrosen und da- 


\begin{tabular}{|c|c|c|}
\hline & Krankheit & Vorkommen \\
\hline \multicolumn{3}{|l|}{ 1. Neonatalzeit } \\
\hline Infektion & $\begin{array}{l}\text { Herpes-, Echo-, Adenoviren, CMV, EBV, Hepatitis } \\
\text { B, andere }\end{array}$ & Häufig \\
\hline Stoffwechselkrankheit & $\begin{array}{l}\text { Fruktoseintoleranz, Galaktosämie, Tyrosinämie } \\
\text { TypI, neonatale Hämochromatose, Morbus } \\
\text { Niemann-Pick Typ C, Atmungskettendefekte, } \\
\text { Mitochondriopathien }\end{array}$ & Selten \\
\hline Ischämie & $\begin{array}{l}\text { Angeborene Herzfehler, Herzchirurgie, Myokardi- } \\
\text { tis, schwere Asphyxie }\end{array}$ & Selten \\
\hline \multicolumn{3}{|l|}{ 2. 4 Wochen bis 3 Jahre } \\
\hline Infektion & $\begin{array}{l}\text { HAV, HBV, NonA-NonB (NonC-NonD), Herpes- } \\
\text { viren, Sepsis, andere }\end{array}$ & Häufig \\
\hline Intoxikation & $\begin{array}{l}\text { Paracetamol, Amanitatoxin (Knollenblätterpilz), } \\
\text { Valproat, Isoniazid, Halothan }\end{array}$ & Selten \\
\hline Stoffwechselkrankheit & Wie 1., $\alpha$-1-Antitrypsinmangel & Selten \\
\hline Ischämie & Wie 1. & Selten \\
\hline Andere & Maligne Krankheiten & Selten \\
\hline \multicolumn{3}{|l|}{ 3. $>3$ Jahre } \\
\hline Infektion & NonA-NonB (NonC-NonD), wie 2. & Häufig \\
\hline Intoxikation & Wie 2. & Selten \\
\hline $\begin{array}{l}\text { Ischämie, Perfusions- } \\
\text { schaden }\end{array}$ & $\begin{array}{l}\text { Budd-Chiari-Syndrom, (veno occlusive disease), } \\
\text { wie } 2 \text {. }\end{array}$ & Selten \\
\hline Stoffwechselkrankheit & Morbus Wilson, $\alpha$-1-Antitrypsinmangel & Selten \\
\hline Andere & $\begin{array}{l}\text { Maligne Krankheiten, Autoimmunhepatitis, } \\
\text { Hyperthermie }\end{array}$ & Selten \\
\hline
\end{tabular}

her prognostisch ungünstig. Die schlechte Lebersyntheseleistung läßt sich an der niedrigen Synthese der Cholinesterase und der Gerinnungsfaktoren erkennen. Die Hyperammonämie ist Ausdruck der beeinträchtigten Entgiftungsfunktion der Leber, korreliert aber nicht immer mit den klinischen Zeichen der Enzephalopathie. Die Blutgasanalyse zeigt ein breites Spektrum von einer metabolischen Acidose bis zur respiratorischen Alkalose. Elektrolytimbalancen sind häufig, ebenfalls Hypoglykämien.

Nierenfunktionsstörung und hepatorenales Syndrom. In den späteren Stadien entwickelt sich häufig eine Niereninsuffizienz, die prognostisch ungünstig zu werten ist. Wegen der verminderten Harnstoffsynthese in der Leber ist der Serumharnstoff nur bedingt als Nierenfunktionsparameter geeignet. Die häufigste Ursache der Niereninsuffizienz ist ein funktionelles Nierenversagen: das hepatorenale Syndrom. Seine Genese ist unklar, charakteristisch ist eine Natriumausscheidung im Urin $<20 \mathrm{mmol} / \mathrm{l}$. Bei der selteneren akuten Tubulusnekrose liegt die Na-Ausscheidung meist $>20 \mathrm{mmol} / \mathrm{l}$. Neben einer sorgfältigen Flüssigkeitsbilanzierung, dem Einsatz von Diuretika unter Überwachung des Elektrolythaushaltes und Dopamin ist die Niereninsuffizienz mit einer kontinuierlichen Hämofiltration effektiv zu behandeln.

Hepatische Enzephalopathie und Hirnödem. Die genaue Pathogenese der Enzephalopathie ist nicht bekannt, es werden verschiedene Hypothesen diskutiert: Veränderung der Blut-Hirn-Schranke, Akkumulation und/oder Synergismus von Neurotoxinen (Ammoniakhypothese), Beeinträchti-
Tabelle 128.1. Ursachen des akuten Leberversagens. (Nach Mowat 1994) gung der Neurotransmission (GABA-Hypothese, falsche Neurotransmitter) und mögliche Verschlechterung des Energiestoffwechsels des Gehirns durch unzureichende Bereitstellung von Substraten (z.B. Glukose). Klinisch wird die Enzephalopathie in 5 Stadien eingeteilt, beginnend mit leichten neuropsychiatrischen Auffälligkeiten (I) über Somnolenz (II), Stupor (III), Koma (IV) bis zum schweren, tiefen Koma (V). Die hohe Letalität des FLV wird maßgeblich durch die Entwicklung eines Hirnödems bestimmt. Als Ursache gelten zum einen ein vasogenes Ödem, bei dem die geschädigte Blut-Hirn-Schranke proteinreiche Flüssigkeit in den Extrazellulärraum entweichen läßt, zum anderen ein intrazelluläres zytotoxisches Ödem. Iatrogene Faktoren können zur raschen Entwicklung eines Hirnödems beitragen (Flüssigkeitsüberladung, anaerober Gehirnstoffwechsel durch nicht korrigierte Hypoglykämie, zerebrale Ischämie durch Hypotension).

Koagulopathie und Blutung. Im Vordergrund steht die verminderte Syntheseleistung von Gerinnungs- und Fibrinolysefaktoren (Faktor I, II, V, VII, IX, X, AT III) in der Leber. Neben einer Thrombopenie sind auch Veränderungen der Thrombozytenmorphologie und -funktion beschrieben. Quick, PTT, Fibrinogen, Faktor II und Faktor V sollten auch nach parenteraler Substitution von Vitamin $\mathrm{K}$ regelmäßig kontrolliert werden. Der Nachweis von Fibrinspaltprodukten ohne Plasminogenaktivator ist Zeichen einer disseminierten intravasalen Gerinnung (DIC). Der Ersatz von Gerinnungs-/Fibrinolysefaktoren sollte in Form von Frischplasma (FFP) nur bei klinischen Blutungszeichen oder bevorstehenden invasiven Maßnahmen (z.B. Legen 


\begin{tabular}{ll}
\hline Stoffklasse & Generika \\
\hline $\begin{array}{l}\text { Nichtsteroidale Antiphlogistika } \\
\text { Antiepileptika }\end{array}$ & $\begin{array}{l}\text { Paracetamol, Acetylsalicylsäure, Indomethazin } \\
\text { Valproinsäure, Carbamazepin, Phenytoin, Phenobarbital }\end{array}$ \\
Narkotika & Halothan, Enfluran, Methoxyfluran \\
Thyreostatika & Propylthiouracil \\
Antibiotika & $\begin{array}{l}\text { Isoniazid, Ketoconazol, Nitrofurantoin, Sulfamethoxazol, } \\
\text { Rifampicin, Tetrazyklin }\end{array}$ \\
Zytostatika & $\begin{array}{l}\text { Methotrexat, 6-Mercaptopurin, Cytosinarabinosid, } \\
\text { Cisplatin, Carboplatin, L-Asparaginase, Cyclophosphamid, } \\
\text { Dactinomycin }\end{array}$ \\
Antihypertonika & $\begin{array}{l}\text { Hydralazin, Methyldopa } \\
\text { Antiarrhythmika }\end{array}$ \\
Antidepressiva/Tranquilanzien & $\begin{array}{l}\text { Amitryptilin, Imipramin, Diazepam, Chlordiazepoxid } \\
\text { Toxine }\end{array}$ \\
& $\begin{array}{l}\text { Amanita-phalloides-Toxin, chlorierte Kohlenwasserstoffe, } \\
\text { Kupfer, Phosphor, Eisensulfat, Lösungsstoffe, Kokain }\end{array}$
\end{tabular}

eines zentralvenösen Katheters) durchgeführt werden. Nach FFP-Gabe muß beachtet werden, daß auch Cholinesterase (CHE) mittransfundiert wird und somit als Lebersyntheseparameter nicht mehr interpretiert werden darf.

\section{Diagnose und Differentialdiagnose}

Infektion. Die häufigste Ursache eines fulminanten Leberversagens im Kindesalter ist mit bis $\mathrm{zu} 80 \%$ eine Virushepatitis. Bei der Hepatitis A ist das Risiko eines FLV mit 0,1-0,4\% sehr niedrig. Die Diagnose wird durch den Nachweis von Anti-HAV-IgM im Patientenserum gesichert. Die Hepatitis B ist in unseren Breiten nicht endemisch, somit ist auch ein durch sie induziertes akutes Leberversagen selten. Die Krankheit wird durch den Nachweis von HBsAg oder Anti-HBc-IgM im Serum diagnostiziert. Bei einer foudroyant ablaufenden Leberzellnekrose kann es aber zu einer raschen Viruselimination kommen, so daß die Diagnose mit konventionellen Hepatitismarkern nicht mehr gesichert werden kann. Mit der Polymerasekettenreaktion (PCR) läßt sich meist auch dann noch der Nachweis von Virus-DNA führen. Neugeborene HBsAg-positiver Mütter, die Anti-HBe-positiv oder weder Anti-HBe- noch Hbe-positiv sind, tragen ein besonders hohes Risiko einer schweren Hepatitis-B-Infektion bis zum akuten Leberversagen. Die Hepatitis $\mathrm{C}$ wird durch den Nachweis von Anti-HCV und HCV-RNA-PCR gesichert, die Hepatitis D durch den Nachweis von Anti-HDV. Beide spielen - wie auch die Hepatitis E - in der Ätiologie des FLV im Kindesalter nur eine untergeordnete Rolle. Die NonANonB-(NonC-NonD)Hepatitis kann nur diagnostiziert werden, wenn die bekannten hepatotropen Viruskrankheiten und andere Hepatopathien ausgeschlossen worden sind. Diese Form der Hepatitis ist die häufigste Ursache eines FLV im Kindesalter in westlichen Ländern. Die spontane Prognose im Kindesalter ist im Vergleich mit anderen Ursachen eines FLV eher ungünstig. Auch nach einer erfolgreichen Lebertransplantation tragen die Patienten ein erhöhtes Risiko, an einer offensichtlich durch die Virusinfektion induzierten aplastischen Anämie zu erkranken. Infektionen mit anderen hepatotropen Viren betreffen vorwiegend das Neugeborenenalter (Herpesviren, Epstein-Barr-Viren, Zytomagalieviren, Adenoviren, Echoviren, Paramyxoviren) und sind mit entsprechenden Antikörperuntersuchungen $\mathrm{zu}$ diagnosti- zieren. Eine Sepsis mit unterschiedlichen Keimen kann auch zu einem FLV führen.

Intoxikation. Die zweithäufigste Ursache eines FLV sind Intoxikationen mit Medikamenten oder Toxinen. Man unterscheidet obligate von fakultativen Hepatotoxinen. Die obligaten Hepatotoxine führen vorhersagbar und dosisabhängig immer zum Leberzellschaden und sind auch im Tierversuch reproduzierbar (z.B. Tetrachlorkohlenstoffvergiftung). Die fakultativen Hepatotoxine induzieren nicht bei jedem Menschen einen Leberzellschaden, die Toxizität ist nicht vorhersagbar, tritt nur sporadisch auf und ist dosisunabhängig. Häufig werden systemische Symptome wie Fieber, Exanthem und eine Eosinophilie beobachtet. Eine mögliche Erklärung dafür ist eine genetisch determinierte atypische Metabolisierung von potentiellen Toxinen, die metabolische Idiosynkrasie. Die bei der Detoxifikation entstehenden reaktiven Metaboliten werden durch einen Stoffwechseldefekt nicht zeitgerecht abgebaut, so daß sie ihre toxische Wirkung frei entfalten können. Bei Halothanintoxikationen konnten in einigen Fällen IgG-Antikörper gegen die Hepatozytenmembran gefunden werden, so daß dieser Mechanismus zur Halothan-induzierten Hepatotoxizität beitragen kann. In Tabelle 128.2 sind die wesentlichsten Medikamente und Toxine aufgeführt, die zu einem FLV führen können.

Eine seltene Ursache der frühkindlichen Leberzirrhose, die sich auch als FLV präsentieren kann, ist die Kupferintoxikation über das Trinkwasser im frühen Säuglingsalter. Die betroffenen Kinder werden mit Milchformula ernährt. Das Wasser für diese Milchnahrung stammt aus einer hauseigenen Brunnenanlage, ist sauer und wird durch Kupferleitungen geführt, aus denen es die Kupferionen löst. Bei dieser Krankheit wird zusätzlich ein genetischer Defekt des Kupferstoffwechsels diskutiert. Möglicherweise kommt es nur bei entsprechend prädisponierten Kindern zu einer Krankheitsmanifestation.

Stoffwechselkrankheiten. Stoffwechselkrankheiten sind genetisch determiniert. Strenggenommen trifft damit die Definition des fulminanten Leberversagens bei Neugeborenen und jungen Säuglingen nicht $\mathrm{zu}$, denn es liegt eine chronische Leberkrankheit vor. Dennoch können sich Stoff- 


\begin{tabular}{|c|c|}
\hline Maßnahme & Ziel \\
\hline Magensonde & $\begin{array}{l}\text { Sichere Verabreichung oraler Medikamente, Magen-pH- } \\
\text { Messung }\end{array}$ \\
\hline Blasenkatheter & Zur Bilanzierung \\
\hline Zentraler Venenkatheter & $\begin{array}{l}\text { Sichere Substitution hochkonzentrierter Lösungen, zentrale } \\
\text { Venendruckmessung, ggf. Dialysezugang }\end{array}$ \\
\hline Arterieller Verweilkatheter & $\begin{array}{l}\text { Blutige Blutdruckmessung, Blutentnahmen, AV-Filtration, } \\
\text { Substitution von Blutprodukten (ARDS-Prophylaxe) }\end{array}$ \\
\hline Epidurale Drucksonde & Monitoring des zerebralen Drucks \\
\hline Intubation und Beatmung; & $\begin{array}{l}\text { Hypoxämie, höhergradige Enzephalopathie (Grad III), } \\
\text { Hirnödem }\end{array}$ \\
\hline Arteriovenöse Filtration & Niereninsuffizienz, unspezifische Giftelimination \\
\hline Hämo- oder Peritonealdialyse & Niereninsuffizienz \\
\hline Plasmapherese & Unspezifische Giftelimination \\
\hline
\end{tabular}

Tabelle 128.3. Invasive Überwachungs- und Therapiemaßnahmen. (Nach Melter et al. 1996) wechselkrankheiten wie die klassische Galaktosämie, die hereditäre Fruktoseintoleranz und die Tyrosinämie Typ I und seltener die neonatale Hämochromatose oder Atmungskettendefekte wie ein FLV präsentieren. Bei der Tyrosinämie gibt es seit einigen Jahren eine neue Behandlungsmethode. Die Produktion wird von toxischen Metaboliten durch eine medikamentöse Blockade des Tyrosinabbaus in einer frühen Stufe durch das Herbizid NTBC verhindert. Diagnostisch wegweisend ist die Ausscheidung entsprechender Metaboliten (Galaktose, Fruktose, Succinylaceton), beweisend sind Enzymaktivitätsbestimmungen, bei der neonatalen Hämochromatose der Nachweis von hohem Serumferritin und der Eisenspeicherung im Gewebe (Wangenschleimhautbiopsat, Leberbiopsat, MRT von Pankreas/Leber). Ab etwa einem Alter von 6 Jahren kann sich ein Morbus Wilson unter dem Bild eines FLV erstmals manifestieren. Das Kupfer im Lebergewebe und im Serum ist charakteristischerweise hoch, das Coeruloplasmin im Serum niedrig, oft ist die alkalische Phosphatase auffallend niedrig. Der homozygote $\alpha$-1-Antitrypsinmangel (PiZZ) führt nur selten zu einem FLV. Der Mangel wird im Serum nachgewiesen, außerdem können Phänotyp und Genotyp bestimmt werden. Andere Stoffwechselkrankheiten wie Zellweger-Syndrom, Alper-Syndrom, Störungen der Fettsäureoxidation, Morbus NiemannPick Typ C und Störungen der oxidativen Phosphorylierung sind sehr seltene Ursachen eines FLV.

Ischämische Hepatopathie. Gegenüber einer Hypoxie ist die Leber sehr resistent. Eine akute oder chronische Herzinsuffizienz kann allerdings auch mit einem akuten Leberversagen einhergehen. Das Budd-Chiari-Syndrom kann zu einem FLV führen, desgleichen die Lebervenenverschlußkrankheit (veno occlusive disease, VOD). Die farbcodierte Dopplersonographie ist bei ischämischen Krankheiten wegweisend.

Andere Ursachen des FLV. Primäre Lebertumoren oder metastatische Lebertumoren können selten ein FLV auslösen, ebenso infiltrative lymphatische Krankheiten oder die hämophagozytotische Lymphohistiozytose. Auch eine Autoimmunhepatitis kann sich initial als FLV manifestieren.

Überwachung, Therapie und Prognose. Wichtige Aspekte der Überwachung und der Therapie sind in den Tabellen 128.3 und 128.4 aufgeführt. Die dort genannten supportiven Maßnahmen dienen lediglich der Zeitgewinnung, um der eigenen Leber die Gelegenheit zur Regeneration zu geben. Die spontane Prognose des FLV im Kindesalter unter rein supportiver Therapie ist mit Überlebensraten von 6-30\% schlecht. Die bisher entwickelten Leberersatzverfahren haben bislang nicht zu einer Verbesserung der Überlebensraten beitragen können. Die Tierleberperfusion (Pavianleber) muß z.Z. noch als experimenteller Therapieansatz gesehen werden, ebenso die Hepatozytenperfusion. Der mehrfach wiederholbare Plasmaaustausch führt zumindest für eine begrenzte Zeit zu einer Stabilisierung des Patienten. In den letzten Jahren wurden in der Entwicklung sog. Bioreaktoren zur Überbrückung einer anhepatischen Phase deutliche Fortschritte gemacht.

Spezifische Therapiemaßnahmen stehen nur für einige Ursachen eines FLV zur Verfügung: Famciclovir oder Lamivudine bei Hepatitis B, Ganciclovir bei CMV- oder EBV-Hepatitis, Aciclovir bei Herpeshepatitis, N-Acetylcystein bei Paracetamolintoxikation, Penicillin und Silibilin bei Knollenblätterpilzvergiftungen und Penicillamin beim Morbus Wilson.

Bei zunehmendem Koma mit Entwicklung eines Hirnödems ist allerdings die Durchführung einer orthotopen Lebertransplantation (OLT) indiziert. Patienten mit einem FLV sollten frühzeitig in ein Zentrum verlegt werden, das das konservative Management beherrscht und wo v.a. auch die Möglichkeit einer OLT gegeben ist. Mit einer Überlebenswahrscheinlichkeit von $60-80 \%$ nach 4 Jahren ist die OLT heute bei entsprechender Indikation die Therapie der Wahl beim fulminantem Leberversagen im Kindesalter.

Eine der schwierigsten Entscheidungen im klinischen Alltag ist die Festlegung des optimalen Zeitpunktes der OLT. Entscheidet man sich zu früh für die OLT, so läuft man Gefahr, einen Patienten zu transplantieren, der evtl. auch ohne OLT eine Restitutio ad integrum erlebt hätte; entscheidet man sich zu spät, kommt es evtl. zu einer Kontraindikation (z.B. irreversibler Hirnschaden), zumindest steigen die Risiken der OLT erheblich an.

In multivariaten Analysen sind verschiedene Versuche unternommen worden, die spontane Prognose des FLV und damit die Notwendigkeit der OLT zu erkennen. Weder die Kings-College-Kriterien (Prothrombinzeit $>100 \mathrm{~s}$, Alter $<10$ Jahre oder Dauer des Ikterus über 1 Woche vor Enzepha- 


\begin{tabular}{|c|c|}
\hline Maßnahme & Indikation/Ziel \\
\hline Flüssigkeitsrestriktion und -bilanzierung & $\begin{array}{l}\text { Prophylaxe und Therapie: Hirnödem, Lungen- } \\
\text { ödem, Aszites }\end{array}$ \\
\hline Reduktion der Proteinzufuhr & Enzephalopathie, Hyperammonämie \\
\hline Verzweigtkettige Aminosäuren & Enzephalopathie, Aminosäurenimbalance \\
\hline $\begin{array}{l}\text { Fruktose- und galaktosefreie Diät } \\
\text { (ggf. nur Reduktion) }\end{array}$ & Sekundäre Fruktose-/Galaktoseintoleranz \\
\hline Laktulose (Ziel: $2-4$ breiige Stühle/Tag) & Darmpassagezeitverkürzung \\
\hline Colistin oder Paromomycin & Selektive Darmdekontamination \\
\hline Amphotericin-B-Suspension & Lokale Pilzprophylaxe \\
\hline Nystatin & Intestinale Pilzprophylaxe \\
\hline Ranitidin, ggf. Omeprazol & $\begin{array}{l}\text { Prophylaxe oberer intestinaler Blutungen, } \\
\text { Magen-pH }>5\end{array}$ \\
\hline Spironolacton & Hyponatriämie, Hypokaliämie, Niereninsuffizienz \\
\hline Furosemid & Niereninsuffizienz, Hyperkaliämie \\
\hline Dopamin & Niereninsuffizienz, Hypotonie \\
\hline Glukoselösung iv (Blutzucker $>5 \mathrm{mmol} / \mathrm{l}$ ) & Hypoglykämie, sek. Hyperinsulinismus \\
\hline Vitamin A, E, K & Vitamin-A-,-E-, -K-Mangel \\
\hline Vermeidung von Sedativa/Anästhetika & Vertiefen hepatisches Koma bzw.Insuffizienz \\
\hline $\mathrm{Na}, \mathrm{Cl}, \mathrm{Ca}, \mathrm{PO}_{4}, \mathrm{~K}, \mathrm{NaHCO}_{3}$ & Imbalancen ausgleichen \\
\hline Humanalbumin ( $\mathrm{NaCl}$-arm) & Hypoproteinämie, Hypotonie \\
\hline FFP (fresh frozen plasma; Frischplasma) & Nur bei Blutungen, invasiven Maßnahmen \\
\hline Thrombozytenkonzentrat & Thrombopenie $<30.000 / \mu$ l oder Blutungen \\
\hline Erythrozytenkonzentrat & Anämie $<8 \mathrm{~g} / \mathrm{dl}$ \\
\hline Somatostatin & Intestinale Blutungen \\
\hline Breite antibiotische Therapie & Bakterielle Infektion, v. a. Sepsis \\
\hline $\begin{array}{l}\text { Amphotericin B + Flucytosin oder } \\
\text { Fluconazol }\end{array}$ & Systemische Pilzinfektion \\
\hline Metamizol & Fieber \\
\hline Mannitol & Hirnödem \\
\hline $\mathrm{N}$-Acetylcystein & Antioxidanzientherapie \\
\hline
\end{tabular}

Tabelle 128.4. Nichtinvasive Überwachungsund Therapiemaßnahmen. (Nach Melter et al. 1996) lopathie oder Gesamtbilirubin $>300 \mu \mathrm{mol} / \mathrm{l}$ und PTT $>50 \mathrm{~s}$ ) noch die Clichy-Kriterien (hepatische Enzephalopathie und Faktor $\mathrm{V}<20 \%$ ) sind an einer größeren pädiatrischen Population validiert worden. Nach eigenen Erfahrungen ist das Ausmaß der Hyperbilirubinämie $(>300 \mu \mathrm{mol} / \mathrm{l})$ und der PTT-Verlängerung (>50 s) prognostisch entscheidend, zusätzliche Parameter sind dialysepflichtige Niereninsuffizienz und ein Leberversagen unklarer Genese. Letztlich muß die Entscheidung zur OLT aber individuell nach sorgfältiger Risikoabwägung und Aufklärung der Eltern getroffen werden. Patienten mit einem FLV werden in einer eigenen höchsten Dringlichkeitsstufe bei der europäischen Transplantationszentrale Eurotransplant gemeldet, so daß es in der Regel möglich ist, innerhalb von $72 \mathrm{~h}$ eine OLT zu realisieren.

Literatur

Bernuau J, Samuel D, Durand F (1991) Criteria for emergency liver transplantation in patients with acute viral hepatitis and factor $\mathrm{V}$ (FV) below $50 \%$ of normal: a prospective study. Hepatology 14: 49a

Lindstedt S, Holme E, Lock EA, Hjalmarson O, Strandvik B (1992) Treatment of hereditary tyrosinaemia type I by inhibition of 4-hydroxyphenylpyruvate dioxygenase. Lancet 340: 813-817

Melter M, Rodeck B, Brodehl J (1996) Akutes Leberversagen im Kindesalter. Monatsschr Kinderheilkd 144: 592-598

Mowat AP (1994) Fulminant and severe acute liver failure. In: Mowat AP (ed) Liver disorders in childhood. Butterworth-Heinemann, Oxford, pp 151-166
Müller T, Feichtinger H, Berger H, Müller W (1996) Endemic Tyrolean infantile cirrhosis: an ecogenetic disorder. Lancet 347: 877-880

O'Grady JG, Williams R (1986) Management of acute liver failure. Schweiz Med Wochenschr 116: 541-544

O'Grady JG, Alexander GJ, Hayllar KM, Williams R (1989) Early indicators of prognosis in fulminant hepatic failure. Gastroenterology 97: 439-445

Pfister ED, Melter M, Rodeck B et al. (1998) Prädiktive Faktoren bei akutem Leberversagen im Kindesalter. Monatsschr Kinderheilkd 146: 98 Sechser A, Osorio J, Freisel C, Osorio RW (2001) Artificial Livar support devices for fulminant livar failure. Clin Liver Dis 5: 415-430

Sokol RJ (1998) Fulminant hepatic failure. In: Balistreri WF, Stocker JT (eds) Pediatric hepatology. Hemisphere, New York, pp 315-362

Whitington PF (1994) Fulminant hepatic failure in children. In: Suchy FJ (edg) Liver disease in children. Mosby, St. Louis, pp 180-213

\subsection{Lebertransplantation}

B. Rodeck

Die Lebertransplantation ist eine Therapieoption bei der terminalen Organinsuffizienz akuter oder chronischer Genese. Sie sollte bei jedem Kind mit einer lebensbedrohenden Leberkrankheit in Betracht gezogen werden. Die 5-Jahresüberlebensraten betragen heute etwa 60-90\% und liegen damit deutlich über den Ergebnissen bei Herz- oder Lungentransplantation. Anders als bei anderen Organtransplantationen erreicht die Überlebenskurve ca. 1 Jahr nach 
Transplantation ein Plateau: Nach dieser Zeit kommt kaum ein Kind transplantationsbedingt zu Tode.

Indikationen. Die häufigste Ursache einer terminalen Leberinsuffizienz im Kindesalter ist eine biliäre Zirrhose, in der Regel auf dem Boden einer extrahepatischen Gallengangatresie. Sie sollte innerhalb der ersten 4-6 Lebenswochen diagnostiziert und mit einer Hepatoportoenterostomie nach Kasai versorgt werden. Etwa 70-80\% der Patienten entwickeln dennoch im weiteren Verlauf eine progrediente biliäre Zirrhose, die zum terminalen Organversagen führt. Aber auch die langfristig nicht erfolgreich operierten Kinder profitieren von der Hepatoportoenterostomie, weil sie damit älter werden als ohne Operation. Außerdem haben sie im Vergleich zu nichtoperierten Kindern eine geringere Dystrophie mit einer besseren Überlebenschance nach der Transplantation. Andere Ursachen cholestatischer Leberkrankheiten mit biliärer Zirrhose sind in der folgenden Übersicht aufgeführt.

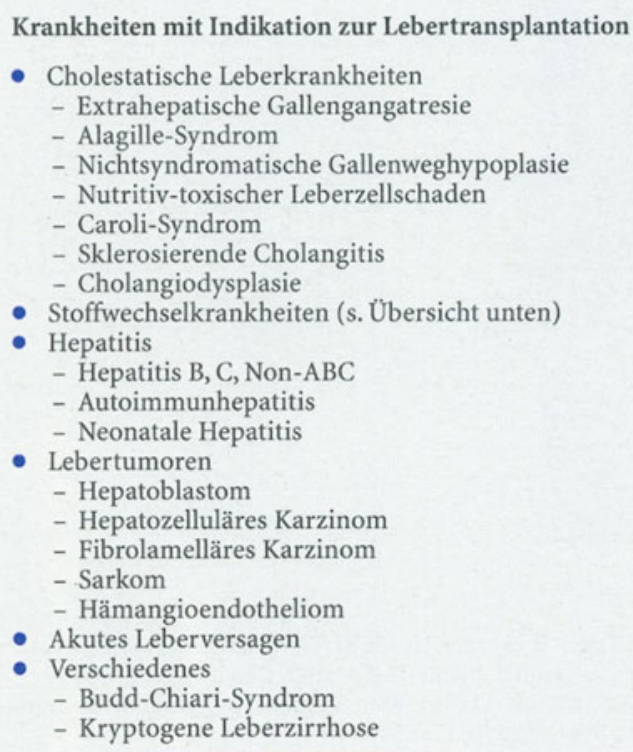

Die zweithäufigste Ursache sind Stoffwechselkrankheiten. Unter ihnen war früher die progressive familiäre intrahepatische Cholestase (PFIC, Morbus Byler) die häufigste. Durch die Entwicklung einer neuen Therapieform, einer externen Galleableitung über eine Cholezystostomie, hat diese Indikation in den letzten Jahren abgenommen. Die partielle externe Galleableitung ist allerdings nur bei Kindern möglich, die noch keine Leberzirrhose entwickelt haben. Andere Stoffwechselkrankheiten gehen entweder mit einer Zirrhose einher oder betreffen nur eine enzymatische Funktion der Leber, die andere Organsysteme des Patienten schwer schädigt, ohne zu einer Zirrhose zu führen (s. Übersicht). Der Indikationsbereich insbesondere der letzten Gruppe hat sich in den letzten Jahren erweitert.

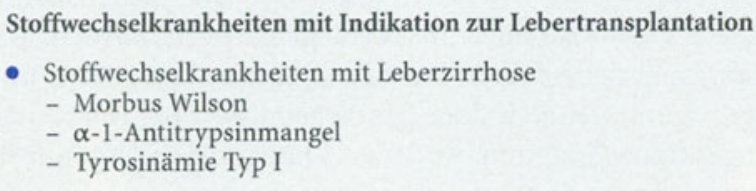

- Galaktosämie

- Neonatale Hämochromatose

- Glykogenose IV

Niemann-Pick-Krankheit

Mukoviszidose

Störungen der Fettsäureoxidation

- Stoffwechselkrankheit ohne Leberzirrhose

- Familiäre Hyperlipidämie

- Primäre Hyperoxalurie

- Crigler-Najjar-Syndrom

- Hämophilie A, B, Protein-C-Mangel

- Harnstoffzyklusdefekte

- Glykogenose Typ I

Im Kindesalter spielen andere Krankheiten wie eine infektiöse Hepatitis, eine Autoimmunhepatitis und die sog. kryptogene Zirrhose nur eine untergeordnete Rolle, häufiger ist ein fulminantes Leberversagen. Schwierig und problematisch ist die Entscheidung, wenn ein Kind mit einem primär inoperablen Lebertumor vorgestellt wird, da auch bei negativer bildgebender Diagnostik Fernmetastasen nicht mit letzter Sicherheit auszuschließen sind und somit ein hohes Risiko des Tumorprogresses besteht.

Insgesamt gibt es aber von seiten der Grundkrankheit keine prinzipielle Kontraindikation, so daß jedes Kind, bei dem das terminale Leberversagen droht, rechtzeitig in einem Lebertransplantationszentrum vorgestellt werden sollte.

Kontraindikationen und Eignungskriterien. Absolute Kontraindikationen sind metastasierende Tumorkrankheiten, nicht beherrschbare Infektionen, vital bedrohende Krankheiten anderer Organsysteme und schwere ZNS-Schäden. Prinzipiell müssen die anatomischen Voraussetzungen für eine Transplantation vorliegen. Eine relative Kontraindikation kann bei einigen Patienten die Ausbildung von pulmonalen arteriovenösen Shunts sein, die mit einer chronischen Hypoxie einhergehen.

Der psychosoziale Hintergrund der Patienten muß präoperativ evaluiert werden. Die Kinder und ihre Familien müssen die Belastung der Transplantation tragen und die immunsuppressive Therapie nach dem Eingriff konsequent durchführen können. Das Transplantationszentrum sollte dementsprechend über eine kompetente auch nichtmedizinische Infrastruktur (Psychologen, Sozialpädagogen, Seelsorger) verfügen.

Die Empfänger werden zentral in der europäischen Transplantationszentrale Eurotransplant im holländischen Leiden erfaßt. Bei Vorliegen eines fulminanten Leberversagens oder eines akuten Transplantatversagens werden die Kinder in einer hochdringlichen Wartestufe geführt, die eine Notfalltransplantation in der Regel innerhalb von 1-2 Tagen ermöglicht. Die Wartezeit auf der normalen Warteliste ist individuell sehr unterschiedlich und kann mehr als $1 \mathrm{Jahr}$ dauern. Eine Alternative stellt die Lebendspendetransplantation dar, bei der ein Elternteil den linkslateralen Leberlappen spendet (s. unten)

Spenderwahl. Leberspender sind Patienten, die nach einem Unfall, einer zerebralen Blutung oder aus anderen Gründen einen Hirntod erleiden. Sie waren zu Lebzeiten mit einer postmortalen Organentnahme einverstanden, oder ihre 
Angehörigen haben nach ihrem Tod einer Organentnahme zugestimmt. Patienten mit HIV-, Hepatitis-B-/-C- oder akuter Varizelleninfektion eignen sich wegen des Risikos der Infektionsübertragung nicht als Organspender. Eine systemische bakterielle Infektion oder Sepsis beim Spender ist eine absolute Kontraindikation. Ausnahmen sind Patienten mit Tod durch Hirnödem nach Meningitis, deren Infektion bereits erfolgreich behandelt wurde. Im Regelfall wird blutgruppengleich (im ABO- und Rhesussystem) transplantiert, nur in Notfallsituationen kann man auch gegen die Blutgruppenbarrieren transplantieren, wobei dann die immunsuppressive Therapie intensiviert werden muß. Eine HLA-Typisierung mit Cross-match wird zwar durchgeführt, spielt aber bei der Entscheidung für oder gegen eine Lebertransplantation keine Rolle.

Operationstechnik. Lebertransplantationen werden orthotop durchgeführt, d.h., die eigene, erkrankte Leber wird komplett entfernt und das Transplantat anatomisch an die Stelle der eigenen Leber eingepflanzt. Die prinzipielle Organknappheit gerade im Kindesalter hat dazu geführt, daß innovative operative Techniken entwickelt wurden, um kleine Kinder mit größenreduzierten Erwachsenenorganen zu versorgen. Dies geschah zunächst mittels der Lebersegmenttransplantation, bei der der linkslaterale Leberlappen eines erwachsenen Spenders in ein Kind transplantiert wurde. Später wurde auch der rechte Lappen zur Transplantation eines erwachsenen Empfängers verwendet, so daß mit einem Spenderorgan zwei Patienten versorgt werden konnten. Daraus entwickelte sich dann die Technik der Lebendspende, bei der einem lebenden Spender (in der Regel einem Elternteil) der linkslaterale Leberlappen reseziert und auf das Kind übertragen wird. Ein Vorteil ist die Planbarkeit der Transplantation ohne lange Wartezeit, allerdings sind bei der Leberresektion potentielle Risiken für den Spender in Kauf zu nehmen. Die Transplantation eines Leberteils bedeutet für die Patienten keinen Nachteil, die Überlebensraten unterscheiden sich nicht von mit kompletten Lebern transplantierten Kindern. Die Organe passen sich in ihrem Wachstum dem des Kindes an.

\section{Postoperatives Management}

Organfunktion. Direkt nach der Transplantation kommt es zu einem mehr oder weniger ausgeprägten Ischämieschaden des Transplantats, der sich meist innerhalb von Tagen zurückbildet. Früh postoperativ kann sich eine initiale Nichtfunktion (INF) des Transplantats herausstellen, die eine rasche Retransplantation erfordert. Die Qualität des Spenderorgans wird präoperativ nach bestimmten Kriterien überprüft. Das Risiko einer INF wird damit minimiert, aber nicht ausgeschlossen. Eine andere Ursache der INF kann eine Thrombose der A. hepatica oder der Pfortader sein.

Akute Abstoßungsreaktionen in den ersten Wochen nach Lebertransplantation sind häufig (40-80\%), später sind sie ausgesprochen selten und meist mit einer zu niedrigen Immunsuppression korreliert. Klinische Symptome können Mattigkeit, Krankheitsgefühl und Fieber sein, oft merken die Patienten aber selbst nichts. Laborchemisch sieht man immer das Korrelat eines Leberzellschadens mit Erhöhung der Transaminasen und der Glutamatlaktatdehydrogena- se (GLDH), gelegentlich eine Cholestase mit Ikterus. Bei Verdacht muß mittels einer Leberbiopsie der histologische Beweis oder Ausschluß geführt werden. Die Therapie erfolgt in Form von hochdosierten Prednisolongaben i.v. $(10 \mathrm{mg} / \mathrm{kg}$ $\mathrm{KG}$ ) über maximal 5 Tage. In der Regel ist die akute Abstoßungsreaktion damit zuverlässig auszubehandeln. Die seltenen steroidresistenten akuten Abstoßungen werden bei mit Cyclosporin A (s. unten) behandelten Patienten mit einer Umstellung auf Tacrolimus (s. unten) therapiert, bei primär mit Tacrolimus behandelten Patienten kann eine Erweiterung der Immunsuppression mit Mycophenolat Mofetil (s. unten) erfolgen. Die Anwendung von Anti-T-LymphozytenGlobulin (ATG) oder Anti-CD3-Antikörpern (OKT3) ist wegen der Gefahr der Entwicklung eines lymphoproliferativen Syndroms in den Hintergrund getreten.

Chronische Abstoßungsreaktionen sind sehr viel seltener, aber auch ungleich schwerer zu behandeln. Klinisch steht hier die Cholestase mit fortschreitender Transplantatzirrhose im Vordergrund. Histologisch findet man ein Verschwinden der kleinen Gallenwege in der Leber (vanishing bile duct syndrome). Fast immer kommt es zum Organverlust. In frühen Stadien kann ein Therapieversuch mit Tacrolimus sinnvoll sein. Bei ausbleibendem Erfolg ist mittelfristig eine erneute Transplantation notwendig.

In einigen Fällen kommt es nach der Transplantation zu hartnäckigen rekurrierenden oder chronischen Cholangitiden. Ursache ist häufig eine Stenose im Bereich der ableitenden Gallenwege. Hier ist eine frühzeitige Diagnostik mittels Sonographie, endoskopischer retrograder Cholangiographie (ERC) oder perkutaner transhepatischer Cholangiographie (PTC) notwendig, um die Situation rasch erkennen zu können und eine entsprechende Therapie (chirurgische Revision, Dilatation über ERC bzw. PTC) einzuleiten. Langfristig ist sonst die Entwicklung einer sekundär sklerosierenden Cholangitis mit Transplantatzirrhose und -verlust zu befürchten.

\section{Medikation nach Lebertransplantation}

Immunsuppression. Nach einer Lebertransplantation ist nach heutigem Kenntnisstand eine lebenslange immunsuppressive Therapie notwendig, um Abstoßungsreaktionen des eigenen Organismus gegen das transplantierte Organ $\mathrm{zu}$ verhindern. Meist wird primär eine Kombination von Cyclosporin A oder Tacrolimus mit Prednisolon eingesetzt. Die Möglichkeiten einer individuellen Immunsuppression sind durch die Entwicklung zusätzlicher Immunsuppressiva (s. unten) in den letzten Jahren deutlich erweitert worden. Zur Zeit werden diese Medikamente in klinischen Studien erprobt. Es ist langfristig zu hoffen, daß zukünftig medikamentös eine Immuntoleranz erzielt werden kann.

Folgende Immunsuppressiva kommen zum Einsatz:

- Prednison: Prednison wird mittel- bis langfristig in einer Dosierung unterhalb der Cushing-Schwellendosis verabreicht, so daß die bekannten Steroidnebenwirkungen zwar nicht erwartet werden, aber doch kontrolliert werden sollten. Mittelfristig wird eine alternierende Therapie mit einer geringen Dosis angestrebt, später ein komplettes Absetzen, um die im Kindesalter gefürchtete Nebenwirkung der Wachstumsretardierung zu vermeiden. 
Tabelle 128.5. Nebenwirkungen von Cyclosporin A und Tacrolimus

\begin{tabular}{|ll|}
\hline Cyclosporin A & Tacrolimus \\
\hline Nephrotoxizität & Nephrotoxizität \\
Neurotoxizität & Neurotoxizität \\
Hepatotoxizität & Hepatotoxizität \\
Hypertonus & Hypertonus \\
Gastrointestinale Symptome & Gastrointestinale Symptome \\
Gingivahyperplasie & Metabolische Acidose \\
Hypertrichose & \\
\hline
\end{tabular}

Tabelle 128.6. Interaktionen von Cyclosporin A und Tacrolimus mit anderen Medikamenten, Erhöhung und Erniedrigung des Vollblutspiegels (Auswahl)

\begin{tabular}{|ll|}
\hline Cyclosporin A & Tacrolimus \\
\hline $\begin{array}{l}\text { Erhöhung des Blutspiegels } \\
\text { Allopurinol }\end{array}$ & \\
Calciumantagonisten & Amoxicillin \\
Cholsäurederivate & Cimetidin \\
Doyxcyclin & Cotrimazol \\
Imidazol-Antimykotika & Cortison \\
Makrolid-Antibiotika & Imidazol-Antimykotika \\
Methylprednisolon & Imipenem \\
Orale Kontrazeptiva & Makrolid-Antibiotika \\
& Nifedipin \\
Erniedrigung des Blutspiegels & Omeprazol \\
Barbiturate & \\
Carbamazepin & Barbiturate \\
Metamizol & Carbamazepin \\
Octeotrid & Dexamethason \\
Phenytoin & Isoniazid \\
Rifampicin & Phenytoin \\
Trimethoprim i.v. & Rifampicin \\
\hline
\end{tabular}

- Cyclosporin A: Die Entwicklung und der klinische Einsatz von Cyclosporin A haben die Transplantationsmedizin in dem heutigen Maß erst ermöglicht. Seine Wirkung beruht auf der Inhibition der Interleukin-2-Produktion auf der Ebene der m-RNA-Bildung in T-Lymphozyten. Cyclosporin A steht intravenös und in einer oral gut resorbierbaren Galenik zur Verfügung. Es wird 2mal am Tag als Lösung oder als Kapsel gegeben. Mittels VollblutTalspiegel-Messungen in EDTA-Blut jeweils $12 \mathrm{~h}$ nach der letzten Einnahme kann die Dosierung individuell eingestellt werden. Der angestrebte therapeutische Bereich in den ersten 6 Monaten nach Transplantation liegt bei etwa $130-250 \mathrm{ng} / \mathrm{ml}$ (gemessen mit monoklonalem RIA), danach bei $80-150 \mathrm{ng} / \mathrm{ml}$.

Die wichtigsten möglichen Nebenwirkungen sind in Tabelle 128.5 aufgeführt. Am häufigsten ist die Nephrotoxizität, die durch verschiedene Medikamente noch verstärkt werden kann (Co-Trimoxazol, Aminoglykoside, Amphotericin B, Ciprofloxacin). Die akute Nephrotoxizität ist nach Dosisreduktion reversibel. Eine häufig auftretende Hypomagnesiämie muß mit einer Substitutionstherapie von Magnesium korrigiert werden. Gastrointestinale Symptome, Hepatoxizität und Neurotoxizität sind eher selten und auch dosisabhängig. Der gelegentliche Hypertonus ist in der Regel gut mit Antihypertensiva einzustellen. Eine Gingivahyperplasie kann durch konsequente Zahnpflege nicht immer verhindert, aber zumindest günstig beeinflußt werden. Eine Hypertrichose stellt ein kosmetisches Problem dar und kann nur mit Enthaarungscremes und Kosmetika behandelt werden. Gelegentlich kann die Hypertrichose auch Grund für ein Umstellen der immunsuppressiven Therapie auf Tacrolimus sein.

Durch Induktion oder Inhibition des Cyclosporinabbaus beeinflussen verschiedene Medikamente den Cyclosporinspiegel und damit auch den Grad der Immunsuppression und der potentiellen Nebenwirkungen (Tabelle 128.6). Diese Medikamente sollten bei mit Cyclosporin behandelten Patienten nur bei strenger Indikationsstellung und unter Kontrolle des Cyclosporin-ASpiegels verordnet werden. Eine entsprechende Anpassung der Cyclosporin-A-Dosierung ist dann notwendig.

- Azathioprin: Ein weiteres gelegentlich eingesetztes Immunsuppressivum ist Azathioprin, das zusammen mit Prednisolon und Cyclosporin A als sog. Tripeltherapie bei Patienten mit rekurrierenden akuten Abstoßungen oder mit Cyclosporin-A-induzierter Nephrotoxizität gegeben wird. In einigen Zentren gehört es auch zu den primär eingesetzten Immunsuppressiva. Die Wirkung beruht auf einer Drosselung der Purinsynthese (DNA und RNA) und einer dadurch bedingten Verzögerung der Lymphozytenproliferation nach Stimulation durch ein Fremdantigen. Die Standarddosierung liegt bei 1-2 mg/ kg. Die wesentliche Nebenwirkung ist die Knochenmarkdepression, bei einer Gesamtleukozytenzahl von <4000/ $\mu \mathrm{l}$ ist die Therapie zu unterbrechen.

- Tacrolimus (FK 506): Tacrolimus hat wohl eine bessere immunsuppressive Wirkung als Cyclosporin. Das Nebenwirkungsspektrum ist sehr ähnlich wie bei Cyclosporin, insgesamt sind Nebenwirkungen aber etwas häufiger. Der Wirkmechanismus ist ähnlich wie bei Cyclosporin A. Einige Zentren setzen Tacrolimus in der primären Immunsuppression in Kombination mit Prednison ein. Andere verwenden es bei steroidresistenten akuten Abstoßungen und immunologischen Risikosituationen. Tacrolimus ersetzt dann die Cyclosporin-A-Therapie. In den ersten Wochen nach Transplantation sollten die Talspiegel zwischen 8 und $12 \mathrm{ng} / \mathrm{ml}$, später zwischen 5 und $10 \mathrm{ng} / \mathrm{ml}$ liegen. Ein für viele Patienten wesentlicher Vorteil ist, daß keine Gingivahyperplasie und nur extrem selten eine Hypertrichose auftritt. Mögliche Nebenwirkungen und Interaktionen mit anderen Medikamenten sind in den Tabellen 121.5 und 121.6 aufgeführt. Letztlich ist ein eindeutiger Voroder Nachteil einer Immunsuppression mit Cyclosporin gegenüber einer Therapie mit Tacrolimus nicht zu belegen, wenngleich sich im Erwachsenenbereich ein leichter Trend zugunsten von Tacrolimus abzeichnet.

- Mycophenolat Mofetil: Mycophenolat Mofetil hemmt die Proliferation von T und B Lymphozyten (spezifische $\mathrm{Pu}-$ rinsynthesehemmung). Es wird wie Azathioprin in Kombination mit Prednison und Cyclosporin A eingesetzt. Eine Interaktion mit anderen Medikamenten ist nicht bekannt. Die Applikation erfolgt oral in Kapselform. Eine Kontrolle der Dosierung ist über Talspiegelbestimmungen möglich, die über $1 \mu \mathrm{g} / \mathrm{mg}$ liegen sollten. Potentielle Nebenwirkungen sind Durchfall, Übelkeit, Erbrechen, Bauchschmerzen, Neutropenie und Thrombopenie. 
- Basiliximab: Basiliximab ist ein monoklonaler chimärer (Maus und Mensch) anti-CD-25 Antikörper mit einer hohen Affinität zum Interleukin-2-Rezeptor. In der frühpostoperativen Phase führt er zu einer Reduktion der Abstoßungsfrequenz in Kombination mit einer Cyclosporin-A-Therapie. Basilixmab wird intravenös appliziert. Es kann wie bei jeder Antikörpertherapie zu allergischen reaktionen kommen.

- Andere Immunsuppressiva: Eine Therapie mit polyklonalem Antilymphozyten-Globulin wie auch mit dem monoklonalen Muronomab-CD3 (OKT3) wird heute nicht mehr durchgeführt, da nach der klinischen Einführung der moderneren Immunsuppressiva das Risiko/ Nutzenverhältnis dieser Antikörper nicht mehr attraktiv ist. Eine Alternative zum Basiliximab ist Daclizumab, ein humaner monoklonaler Interleukin-2-Rezeptor-Antikörper. Der Zytokin-Inhibitor Sirolimus kann in der Kombination mit den Calzineurininhibitoren Cyclosporin A oder Tacrolimus als Reservemedikament z.B. bei Nephrotoxizität eingesetzt werden.

Mycophenolat Mofetil, Basiliximab und die genannten neuen Immunsuppressiva werden z.Zt. im Rahmen von klinischen Studien eingesetzt. Das Arsenal der heute zur Verfügung stehenden Immunsuppressiva führt dazu, daß die Therapie individualisiert werden kann und die Patienten je nach eigenem immunologischen Profil eingestellt werden können.

Eine generelle Nebenwirkung der immunsuppressiven Therapie ist ein erhöhtes Malignomrisiko. Hier sind in erster Linie Epstein-Barr-Virus-induzierte B-Zell-Lymphome zu nennen, bei Erwachsenen findet man eine erhöhte Inzidenz von Hauttumoren (Basaliome, Spinaliome). Eine weitere Nebenwirkung ist die erhöhte Anfälligkeit insbesondere gegenüber Virusinfektionen der Herpesvirusgruppe.

Andere Medikamente. Eine diuretische Therapie zur Ausschwemmung bzw. Prophylaxe von Aszites ist in der Regel nur in der unmittelbaren postoperativen Phase maximal 3-6 Monate nach Transplantation notwendig. Wegen der geringen Lumina der Gefäße und Gefäßanastomosen im Kindesalter sollte eine Thrombozytenaggregationshemmung mit Acetylsalicylsäure bei Kindern unter $15 \mathrm{~kg} \mathrm{KG}$, in einzelnen Fällen auch bei schwereren Kindern, über den Zeitraum von 1 Jahr durchgeführt werden, um Gefäßthrombosen insbesondere der A. hepatica zu verhindern.

Infektion. Die häufigste Todesursache nach Lebertransplantation sind Infektionen. Bakterielle und Pilzinfektionen sind Ursache der relativ hohen frühpostoperativen Morbidität und Letalität. Im Langzeitverlauf sind sie wesentlich seltener und in der Regel problemlos zu therapieren. Hier sind die Virusinfektionen insbesondere der Herpesvirusgruppe (Varicella-Zoster-, Herpes-simplex-, Zytomegalie-, EpsteinBarr-Virus) ein größeres Problem. Bei Windpockenkontakt eines immunsupprimierten, bislang nicht erkrankten oder geimpften Kindes sollte eine Prophylaxe mit Aciclovir durchgeführt werden, bei Erkrankung eine intravenöse Therapie. Herpes-simplex-Krankheiten werden je nach Schweregrad lokal oder systemisch mit Aciclovir behandelt. Eine Zytomegaliekrankheit verläuft von der klinischen Symptomatologie her uncharakteristisch, eine hohe Fieberzacke am Tag neben allgemeinem Krankheitsgefühl kann Leitsymptom sein. Häufig verläuft die Krankheit als Hepatitis. Bei Beweis ist eine intravenöse Therapie mit Ganciclovir indiziert. Bei unkompliziertem Verlauf ist auch eine orale Therapie möglich. Auf die Gefahr einer durch eine EBV-Infektion induzierten lymphoproliferativen Krankheit wurde schon eingegangen. Banale Infekte der oberen Luftwege sind ähnlich häufig wie in der Normalbevölkerung und verlaufen unter der Immunsuppression nicht schwerer.

Impfungen. Die Immunantwort nach aktiven Impfungen bei immunsupprimierten Patienten ist schlecht. Daher sollten Kinder, bei denen eine Lebertransplantation absehbar ist, möglichst schon präoperativ komplett durchgeimpft werden (DTP, Polio, MMR, HIB, Varizellen, Pneumokoken, Hepatitis A, Hepatitis B, ggf. FSME). Postoperativ ist der jeweilige Impferfolg auf jeden Fall durch entsprechende serologische Kontrollen zu überprüfen. Totimpfstoffe können ohne Bedenken verabreicht werden. Lebendimpfungen sind prinzipiell kontraindiziert. Eine Ausnahme ist die Varizellenimpfung, die etwa 1 Jahr nach Transplantation durchgeführt werden kann, wenn die Immunsuppression in der Regel niedriger dosiert ist. Gegenüber passiven Impfungen bestehen keinerlei Kontraindikationen.

Lebensqualität. Die medizinische Rehabilitation lebertransplantierter Kinder ist in der Regel gut. Die meisten Patienten zeigen nach erfolgreicher Transplantation ein Aufholwachstum bei oft vorher bestehendem Minderwuchs. Die Compliance ist im jungen Kindesalter bei in der Regel hoch motivierten Eltern ebenfalls generell gut, Probleme treten aber zunehmend in und nach der Pubertät auf. Hier ist eine sorgfältige, kompetente und engagierte Führung der jungen Patienten unbedingt erforderlich, um das Organ zu erhalten. Die Kinder sollen nach Organtransplantation möglichst ungehindert altersgerecht aufwachsen, eine Einschränkung ihrer Sozialkontakte ist keinesfalls notwendig. Der Besuch von Kindergarten und Schule ist ohne Beschränkung möglich. Bei der Berufswahl ist die lebenslang notwendige immunsuppressive Therapie zu bedenken. Auch Schwangerschaften sind nach einer komplikationslosen Transplantation möglich.

\section{Literatur}

Bismuth H, Houssin D (1984) Reduced-sized orthotopic liver graft in hepatic transplantation in children. Surgery 95: 367-370

Broelsch CE, Emond JC, Whitington PF, Thistlethwaite JR, Baker AL Lichtor JL (1990) Application of reduced-size liver transplants as split grafts, auxiliary orthotopic grafts, and living related segmental transplants. Ann Surg 212:368-375

Koverik JM, Gridelli BG, Martin S et al. (2002) Basiliximab in pediatric liver tranplantation: a pharmacokinetic-derived dosing algorithm. Pediatr Transplant 6: 224-230

Lee H, Vacanti JP (1996) Liver transplantation and its long-term management in children. Pediatr Clin North Am 43: 99-124

Martinez Ibanez V, Boix Ochoa J, Lloret J, Broto J (1992) Paediatric liver transplantation: life after portoenterostomy in biliary atresia. J Pediatr Surg 27: 830-832

McDiarmid SV, Busuttil RW, Ascher NL et al. (1995) FK506 (tacrolimus) compared with cyclosporine for primary immunosuppression after pediatric liver transplantation. Results from the U.S. Multicenter Trial. Transplantation 59: 530-536

Pichlmayr R, Ringe B, Gubernatis G, Hauss J, Bunzendahl H (1988) Transplantation einer Spenderleber auf zwei Empfänger (Splitting 
Transplantation) - eine neue Methode in der Weiterentwicklung der Lebersegmenttransplantation. Langenbecks Arch Chir 373: 127-130

Pruim J, Klompmaker IJ, Haagsma EB, Bijleveld CMA, Slooff MJH (1993) Selection criteria for liver donation: a review. Transpl Int 6: 226-235

Ryckman FC, Ziegler MM, Pedersen SH et al. (1994) Liver transplantation in children. In: Suchy FJ (ed) Liver disease in children. Mosby, St. Louis, pp 930-950
Rodeck B, Melter M, Kardorff R et al. (1996) Liver transplantation in children with chronic end stage liver disease: factors influencing survival after transplantation. Transplantation 62: 1071-1076

Rodeck B, Melter M, Kardorff R et al. (1996) Lebertransplantation im Kindesalter. Monatsschr Kinderheilkd 144: 490-495

\section{Budd-Chiari-Syndrom}

\section{Burdelski}

Diese im Kindesalter nur selten zu beobachtende Krankheit besteht in einer Obstruktion der Lebervenenausflußbahn. Sie kann sich auf 3 Ebenen abspielen: intrahepatisch, in den Lebervenen und in der suprahepatischen V. cava inferior. Je nach Lokalisation der Obstruktion müssen unterschiedliche Ursachen in Betracht gezogen werden.

Intrahepatische Obstruktion. Diese Form der Lebervenenobstruktion wird am häufigsten nach Knochenmarktransplantation beobachtet. Sie wird als „veno occlusive disease“ (VOD) bezeichnet. Histologisch findet sich eine konzentrische Einengung der terminalen Venolen. Obstruktionen im Bereich der Lebervenen und der V. cava liegen nicht vor. Das Spektrum der Ursachen dieser Krankheit außerhalb der Knochenmarktransplantation ist breit. Es reicht von Alkaloiden und Aflatoxinen in der Nahrung über medikamentös ausgelöste Formen durch Hypervitaminosen, Chemotherapeutika, Immunsuppressiva, Cysteamin und Polysorbat über Bestrahlung bis hin zu Thrombophilien, Polyzythämie, lymphoproliferativen Krankheiten, Tumorinvasion, chronisch-entzündlichen Darmkrankheiten, Sichelzellanämie, Kollagenosen und Immundefekten (Tabelle 129.1).

Die klinischen Symptome dieser Form der Krankheit sind plötzliches Auftreten von Aszites und Hepatomegalie, die $\mathrm{zu}$ aufgetriebenem Abdomen, Bauchschmerz und rasch zunehmender Splenomegalie führen. Die Diagnose wird per Ultraschall in Kombination mit der Dopplersonographie gestellt. Beim Erwachsenen stellt inzwischen die Anlage eines transjugulären intrahepatischen portosystemischen Shunts (TIPS) eine Behandlungsoption vor einer Lebertransplantation dar. Sie wird bei größeren Kindern auch eingesetzt werden können; bei kleineren Kindern ist dies zu bezweifeln, da die Größe der Schleuse durch das Lumen der V. jugularis interna eingeschränkt ist. Bei zunehmender Leberinsuffizienz ist die Lebertransplantation die Therapie der Wahl: Thrombophilien als Ursache der Krankheit werden sogar kausal behandelt, bei den übrigen Ursachen muß neben der immunsuppressiven Therapie auch die Grundkrankheit behandelt und eine Antikoagulanzientherapie langfristig durchgeführt werden.

Obstruktion der Lebervenen. Bei dieser Lokalisation finden sich angeborene Fehlbildungen der Lebervenen.
Tabelle 129.1. Ursachen der Lebervenenverschlußkrankheit (Auswahl)

\begin{tabular}{|ll|}
\hline Ursache & Beispiel \\
\hline $\begin{array}{l}\text { Fehlbildungen } \\
\text { Immundefekte }\end{array}$ & Membranen, Hypoplasien \\
Hämatologische Krankheiten & Thrombozythämie, Thrombophilie \\
Nahrungsmittel & Alkaloide, Aflatoxine \\
Tumoren & Wilms-Tumor, lymphoproliferative \\
Kedikamente & $\begin{array}{l}\text { Krankheiten } \\
\text { Vitamine, Zytostatika, Immun- } \\
\text { suppressiva, Antikonzeptiva }\end{array}$ \\
Unklare Ursachen & Kollagenosen, Morbus Crohn \\
\hline
\end{tabular}

Obstruktion der suprahepatischen V. cava inferior. Diese Obstruktion der Lebervenenausflußbahn ist weltweit die häufigste, allerdings ist sie überwiegend in Entwicklungländern anzutreffen. Trotz der offensichtlich angeborenen Läsionen werden die meisten Patienten ohne inzidentelle Ultraschalluntersuchung erst im Erwachsenenalter diagnostiziert. Unter diesen Bedingungen besteht ein deutlich erhöhtes Risiko für ein hepatozelluläres Karzinom.

Die klinischen Symptome dieser Form sind unterschiedlich, sie können entweder schleichend ohne initiale Aszitesbildung verlaufen oder aber als akute zentrolobuläre Nekrose mit dem Erscheinungsbild des akuten Leberversagens auftreten. Differentialdiagnostisch muß eine Pericarditis constrictiva ausgeschlossen werden. Auch bei diesen Formen des Budd-Chiari-Syndroms ist die Lebertransplantation die Therapie der Wahl.

Literatur

Gentil-Kocher S, Bernard O, Brunelle F et al. (1988) Budd-Chiari syndrome in children: report of 22 cases. J Pediatr 113: $30-38$

Ludwig J, Hashimoto E, McGill DB et al. (1990) Classification of hepatic venous outflow obstruction: ambiguous terminology of the BuddChiari syndrome. Mayo Clin Proc 65: 51-55

McDonald GB, Sharma P, Mattews DE et al. (1984) Veno-occlusive disease of the liver after bone marrow transplantation: diagnosis, incidence and predisposing factors. Hepatology 4: 116-122

Rössle M, Siegerstetter V, Huber M et al. (1998) The first decade of the transjugular intrahepatic portosystemic shunt (TIPS): state of the art. Liver 18: 73-89 